\title{
Commensal discovery of four fast radio bursts during Parkes Pulsar Timing Array observations
}

\author{
S. Osłowski ${ }^{\oplus},{ }^{1 \star}$ R. M. Shannon, ${ }^{1}$ V. Ravi, ${ }^{2}$ J. F. Kaczmarek ${ }^{\oplus},{ }^{3}$ S. Zhang,,${ }^{4,3,5,6}$ \\ G. Hobbs ${ }^{\oplus},{ }^{3}$ M. Bailes, ${ }^{1}$ C. J. Russell, ${ }^{3}$ W. van Straten ${ }^{\oplus}{ }^{7}$ C. W. James ${ }^{\odot}, 8$ \\ A. Jameson, ${ }^{1}$ E. K. Mahony, ${ }^{3}$ P. Kumar ${ }^{\oplus},{ }^{1}$ I. Andreoni ${ }^{\oplus,},{ }^{2,1}$ N. D. R. Bhat, ${ }^{8}$ \\ S. Burke-Spolaor, ${ }^{9,10}$ S. Dai, ${ }^{3}$ J. Dempsey, ${ }^{11}$ M. Kerr ${ }^{\oplus},{ }^{12}$ R. N. Manchester, ${ }^{3}$ \\ A. Parthasarathy ${ }^{\oplus},{ }^{1}$ D. Reardon ${ }^{\odot},{ }^{1}$ J. M. Sarkissian, ${ }^{3}$ R. Spiewak, ${ }^{1}$ L. Toomey, ${ }^{3}$ \\ J.-B. Wang, ${ }^{13}$ L. Zhang ${ }^{14,5,3}$ and X.-J. Zhu ${ }^{\oplus 15}$ \\ ${ }^{1}$ Centre for Astrophysics and Supercomputing, Swinburne University of Technology, PO Box 218, Hawthorn, VIC 3122, Australia \\ ${ }^{2}$ Cahill Center for Astrophysics, California Institute of Technology, Pasadena, CA 91125, USA \\ ${ }^{3}$ CSIRO Astronomy and Space Science, Australia Telescope National Facility, Box 76, Epping, NSW 1710, Australia \\ ${ }^{4}$ Purple Mountain Observatory, Chinese Academy of Sciences, Nanjing 210008, China \\ ${ }^{5}$ University of Chinese Academy of Sciences, Beijing 100049, China \\ ${ }^{6}$ International Centre for Radio Astronomy Research, University of Western Australia, Crawley, WA 6009, Australia \\ ${ }^{7}$ Institute for Radio Astronomy and Space Research, Auckland University of Technology, Private Bag 92006, Auckland 1142, New Zealand \\ ${ }^{8}$ International Centre for Radio Astronomy Research, Curtin University, Bentley, WA 6102, Australia \\ ${ }^{9}$ Department of Physics and Astronomy, West Virginia University, PO Box 6315, Morgantown, WV 26506, USA \\ ${ }^{10}$ Center for Gravitational Waves and Cosmology, West Virginia University, Chestnut Ridge Research Building, Morgantown, WV 26505, USA \\ ${ }^{11}$ CSIRO Information Management and Technology, GPO Box 1700, Canberra, ACT 2601, Australia \\ ${ }^{12}$ Naval Research Laboratory, 4555 Overlook Ave., SW, Washington, DC 20375, USA \\ ${ }^{13}$ Xinjiang Astronomical Observatory, Chinese Academy of Sciences, 150 Science 1-Street, Urumqi, Xinjiang 830011, China \\ ${ }^{14}$ National Astronomical Observatories, Chinese Academy of Sciences, A20 Datun Road, Chaoyang District, Beijing 100101, China \\ ${ }^{15}$ School of Physics and Astronomy, Monash University, Clayton, VIC 3800, Australia
}

Accepted 2019 June 21. Received 2019 June 20; in original form 2019 May 16

\begin{abstract}
The Parkes Pulsar Timing Array (PPTA) project monitors two dozen millisecond pulsars (MSPs) in order to undertake a variety of fundamental physics experiments using the Parkes 64-m radio telescope. Since 2017 June, we have been undertaking commensal searches for fast radio bursts (FRBs) during the MSP observations. Here, we report the discovery of four FRBs $(171209,180309,180311$, and 180714). The detected events include an FRB with the highest signal-to-noise ratio ever detected at the Parkes Observatory, which exhibits unusual spectral properties. All four FRBs are highly polarized. We discuss the future of commensal searches for FRBs at Parkes.
\end{abstract}

Key words: methods: data analysis - methods: observational-radio continuum: general.

\section{INTRODUCTION}

Fast radio bursts (FRBs) are millisecond-duration radio flashes of unknown origin. They were first discovered during the reprocessing of archival data from a pulsar survey of the Magellanic Clouds (Lorimer et al. 2007). Currently, there are a few tens of FRBs known (Petroff et al. 2016, http://frbcat.org/). Most of these have only been detected once. However, the 'repeating' FRBs 121102 (Spitler et al. 2016) and 180814 (CHIME/FRB Collaboration et al. 2019b) have been detected on multiple occasions. The first repeating FRB has

\footnotetext{
^E-mail: stefanoslowski@swin.edu.au
}

been localized to a dwarf galaxy at redshift of 0.193 (Chatterjee et al. 2017; Tendulkar et al. 2017). Although the remaining FRBs have not been localized, there is evidence for their extragalactic origin, primarily that the integrated electron column density for these FRBs is well in excess of the expected Galactic contribution along the line of sight. While the majority of the bursts have been detected at medium $(>19.5)$ or high $\left(>42^{\circ}\right)$ Galactic latitudes, FRBs have also been detected at low Galactic latitudes. Recently, Bhandari et al. (2018) concluded that there is no strong evidence for a dependence of the FRB rate with latitude despite early indications that there was (Burke-Spolaor \& Bannister 2014; Petroff et al. 2014).

FRBs promise to be probes of the intergalactic medium (IGM) and independent cosmological probes (e.g. McQuinn 2014; Fi- 
alkov \& Loeb 2016), although some authors doubt the usefulness of FRBs for more novel cosmological tests (Jaroszynski 2019). By analysing FRB dispersion measures (DMs) together with models for the host galaxy and Milky Way interstellar medium, important insight can be gained into the baryon densities in the circumgalactic and intergalactic medium (Prochaska \& Zheng 2019; Ravi et al. 2019). Furthermore, if an FRB is polarized, we can determine the Faraday rotation providing information on the magnetic field along the line of sight.

The Parkes Pulsar Timing Array (PPTA; Manchester et al. 2013) is a project in which a sample of 22 millisecond pulsars (MSPs) spread across the celestial sphere are observed using the Parkes 64 $\mathrm{m}$ radio telescope. The primary goals are to detect low-frequency gravitational waves (Shannon et al. 2015), errors in the Solar system ephemeris (Champion et al. 2010), and instabilities in atomic timescales (Hobbs et al. 2012). The PPTA data sets also enable studies of individual pulsars (e.g. Dai et al. 2015). The observations occur at roughly fortnightly cadence at three wavelengths. Until recently, the $20 \mathrm{~cm}$ observations were primarily obtained using the central beam of a 13-beam multibeam receiver (Staveley-Smith et al. 1996). In 2017 June, we commenced searching all of the 13 beams in near realtime for FRB events. An advantage of a commensal search during a programme that repeatedly looks at the same sky location is that we are both able to search for FRBs and quantify the repeatability of any detected FRB.

This work summarizes the results of our commensal FRB search so far, the first of its kind. Within $1 \mathrm{yr}$, we have found four FRBs. We summarize our observations in Section 2. Section 3 describes the basic characteristics of the FRBs we found. In Section 4, we discuss various implications of our discoveries, before concluding in Section 5.

\section{OBSERVATIONS AND ANALYSIS}

During standard PPTA observations, we observe 22 MSPs at roughly fortnightly cadence with occasional observations of three additional lower priority pulsars. Roughly half the observing time is spent using the dual-band coaxial ' $10 \mathrm{~cm} / 50 \mathrm{~cm}$ ' receiver (Granet et al. 2005), while during the rest we use the multibeam receiver. The observations discussed in this paper were all recorded with the latter receiver. The receiver provides 13 beams with sky separations of approximately 29 arcmin, and we always point the telescope such that the target pulsar is in the central beam of the receiver. Note that for PPTA observing we do not ensure that the parallactic angle of the receiver is held constant during the observation, so the non-central beams do not always point at exactly the same sky positions over the duration of an observation.

All of the MSPs are within our Galaxy (note that one of our sources, PSR J1824-2452A, is associated with the M28 globular cluster). Table 1 shows the properties of the pulsars relevant to this work, in that we were observing these pulsars with the receiver's centre beam at the time of FRB detection. The table columns give

Table 1. Key properties of the pulsars relevant to this work.

\begin{tabular}{lccccr}
\hline PSR & $\begin{array}{c}P(\mathrm{~ms}) \\
(\mathrm{ms})\end{array}$ & $\begin{array}{c}\mathrm{DM} \\
\left(\mathrm{cm}^{-3} \mathrm{pc}\right)\end{array}$ & $\begin{array}{c}\mathrm{RM} \\
\left(\mathrm{rad} \mathrm{m}^{-2}\right)\end{array}$ & $\begin{array}{c}S \\
(\mathrm{mJy})\end{array}$ & \multicolumn{1}{c}{$\begin{array}{c}b \\
\left({ }^{\circ}\right)\end{array}$} \\
\hline $\mathrm{J} 1545-4550$ & 3.575 & 68.39 & 6.10 & 0.75 & 6.988 \\
$\mathrm{~J} 1744-1134$ & 4.075 & 31.137 & 2.2 & 13 & 9.180 \\
$\mathrm{~J} 2124-3358$ & 4.931 & 4.60 & -0.40 & 3.60 & -45.438 \\
$\mathrm{~J} 2129-5721$ & 3.726 & 31.85 & 22.30 & 1.10 & -45.570 \\
\hline
\end{tabular}

the pulse period $(P), \mathrm{DM}$, rotation measure $(\mathrm{RM})$, mean flux density at the frequency of $1400 \mathrm{MHz}(S)$, and Galactic latitude $(b)$, as per the Australia Telescope National Facility (ATNF) Pulsar Catalogue (Manchester et al. 2005). ${ }^{1}$

When observing in the $20-\mathrm{cm}$ band, we use two backends: the fourth generation of Pulsar Digital Filter Bank (PDFB4) and the CASPER $^{2}$ Parkes Swinburne Recorder (CASPSR). These backends are only used to record the data from the central beam in a data format that is in general not suitable for searching for transient events (the data streams are folded at the known period of the observed pulsar). In 2017 June, we have enabled the remaining 12 beams and performed a search for transient events in all 13 beams. We use a real-time search process nearly identical to that of the SUrvey for Pulsars and Extragalactic Radio Bursts (SUPERB) project's 'Fast' pipeline (see description in Keane et al. 2018), which itself is an evolution of an older pipeline (Keith et al. 2010). Here we only summarize the key elements of the pipeline. The pipeline uses the multibeam receiver and the Berkeley Parkes Swinburne Recorder (BPSR). The dual polarization 8-bit data stream from all 13 beams is stored in a ring buffer over the full available bandwidth of $400 \mathrm{MHz}$ centred at $1382 \mathrm{MHz}$ and channelized into 1024 channels, each sampled at a rate of $15.625 \mathrm{kHz}$ (corresponding to time resolution of $64 \mu \mathrm{s}$ ). The data are decimated and averaged to form a 8-bit total intensity filter bank, which is searched using the HEIMDALL ${ }^{3}$ (Barsdell 2012) software up to a maximum DM of $4096 \mathrm{~cm}^{-3} \mathrm{pc}$ with the number of trials determined by setting acceptable loss of signal-to-noise ratio $(\mathrm{S} / \mathrm{N})$ to be up to 20 per cent of that at the optimal DM. The pipeline automatically determines if a transient candidate is a potential FRB based on a number of factors. These include the final $\mathrm{S} / \mathrm{N}$ of processed data, as well as the discovery signal-to-noise ratio as reported by HEIMDALL $\left(\mathrm{S} / \mathrm{N}_{\mathrm{H}}\right)$, width of the transient, the number of events around the time of the event, and ratio of DM to the maximum contribution from our own Galaxy along the line of sight. If a candidate has satisfactory values for all the aforementioned parameters (see equation 1 in Bhandari et al. 2018), we temporarily store a full-polarization 8-bit version of the filter bank for offline analysis. The data set is available from the CSIRO pulsar data archive (Osłowski et al. 2018a).

If the automated pipeline identifies a likely FRB candidate, it notifies the observers in a live monitoring tool and via email by providing a number of diagnostic plots and metadata. Based on these, a team member decides whether the event is likely to be a real astrophysical source. In contrast with SUPERB's strategy, we do not run any offline search pipeline. If the team member believes that the source is credible, then we ensure the 8-bit full Stokes data are permanently retained for subsequent analysis. We remove narrowband radio frequency interference (RFI) by applying a median filter, i.e. comparing the total flux density in each channel with that of its 49 neighbouring channels. We do not perform any automated mitigation of impulsive interference, which can be detected as lowDM transient candidates.

The multibeam receiver is equipped with a noise diode that is coupled to the receptors and driven with a square wave to inject a polarized reference signal into the feed horn. This signal is typically recorded for 2 min before every observation of a pulsar. The observation of the noise diode allows estimation of and

\footnotetext{
${ }^{1}$ Version 1.60, http://www.atnf.csiro.au/people/pulsar/psrcat/.

${ }^{2}$ Center for Astronomy Signal Processing and Electronics Research at University of California, Berkeley.

${ }^{3}$ http://sourceforge.net/projects/heimdall-astro/
} 
Table 2. Observed and inferred properties of the FRBs discovered during PPTA observations. All properties are model dependent.

\begin{tabular}{|c|c|c|c|c|}
\hline Property & FRB 171209 & FRB 180309 & FRB 180311 & FRB 180714 \\
\hline Beam number & 13 & 1 & 4 & 7 \\
\hline$l, b\left(^{\circ}\right)$ & $332.3,6.2$ & $10.9,-45.4$ & $337.4,-43.7$ & $14.9,8.7$ \\
\hline $\mathrm{S} / \mathrm{N}$ & 40 & 411 & 11.5 & 22 \\
\hline $\mathrm{S} / \mathrm{N}_{\mathrm{H}}$ & 35.8 & 112.8 & 15.3 & 19.8 \\
\hline $\mathrm{DM}\left(\mathrm{cm}^{-3} \mathrm{pc}\right)$ & $1457.4 \pm 0.03$ & $263.42 \pm 0.01$ & $1570.9 \pm 0.5$ & $1467.92_{-0.2}^{+0.3}$ \\
\hline Target PSR & $\mathrm{J} 1545-4550$ & $\mathrm{~J} 2124-3358$ & J2129-5721 & $\mathrm{J} 1744-1134$ \\
\hline$L_{\mathrm{f}}$ & $1.00 \pm 0.01$ & $0.4556 \pm 0.0006$ & $0.75 \pm 0.03$ & $0.91 \pm 0.03$ \\
\hline$V_{\mathrm{f}}$ & $0.00 \pm 0.01$ & $0.2433 \pm 0.0005$ & $0.11 \pm 0.02$ & $0.05 \pm 0.02$ \\
\hline $\mathrm{DM}_{\mathrm{gal}}\left(\mathrm{cm}^{3} \mathrm{pc}\right)$ & 235 & 30 & 32 & 223 \\
\hline$\tau(\mathrm{ms})$ & $0.138_{-0.013}^{+0.015}$ & $0.086_{-0.0008}^{+0.0006}$ & $1.45_{-0.23}^{+0.25}$ & $0.38_{-0.6}^{+0.08}$ \\
\hline$\tau_{\mathrm{DM}}(\mathrm{ms})$ & 2.86 & 0.52 & 3.08 & 2.88 \\
\hline
\end{tabular}

correction for the polarization impurity. We note that, during normal observations, we only undertake careful modelling of polarimetry and sensitivity of the central beam of the receiver and thus the uncertainties on the measured properties of events occurring in noncentral beams can be larger than that typical for pulsar observations at Parkes. We verified our calibration procedure to the first order by observing bright well-known pulsars and placing them in the non-central beams of the multibeam receiver, including in positions offset from the beam centre. A similar procedure was adopted by Caleb et al. (2018) who also concluded that the polarimetry of BPSR is reliable to the first order.

After calibrating the data for the FRB candidates, we performed a search for Faraday rotation by maximizing the $\mathrm{S} / \mathrm{N}$ of the linear polarization as implemented in the RMFIT tool provided as part of the PSRCHIVE software suite (Hotan, van Straten \& Manchester 2004; van Straten, Demorest \& Osłowski 2012); see e.g. Han et al. (2006) for more details. After obtaining the RM spectrum from RMFIT the central values were refined by fitting a Gaussian function in cases where the spectrum showed complex features. The observed properties of the FRBs, such as the width, scattering parameters, and DM, were determined as described in Ravi (2019). For each FRB we fitted all the models described there, as well as an additional model that composed of a burst with an intrinsic width, and scattering with its frequency dependence as an extra free parameter. We chose the best model based on the approximate Bayes factor, i.e. the Bayesian information criterion (Schwarz 1978), and we adopted a threshold of 3 to select a more complex model.

\section{RESULTS}

We found four FRBs that were initially reported as Astronomical Telegrams (Shannon et al. 2017; Osłowski et al. 2018b,c,d). Table 2 summarizes their observed and inferred, model-dependent properties. In the table, RA and Dec. denote the right ascension and declination of the centre of the beam of the detection ${ }^{4}$ at the time of the burst, respectively. $l$ and $b$ are the Galactic longitude and latitude in degrees and $\mathrm{DM}_{\mathrm{gal}}$ is the Galactic contribution to the DM as provided by the 'YMW16' model (Yao, Manchester \& Wang

${ }^{4}$ All FRBs have positional uncertainty of a circle with 7.5 arcmin radius.
2017). $\tau$ is the scattering time in milliseconds at the frequency of $1 \mathrm{GHz}, \tau_{\mathrm{DM}}$ is the DM broadening in a single channel at the bottom of the band, and $W_{\mathrm{i}}$ is the intrinsic width of the pulse, if measurable. $F$ is the fluence estimate from the radiometer equation, and the redshift limit, $z$, as provided by the YMW16 model.

We note that both the DM and RM values, when measured, for our FRBs are significantly different than these quantities for the pulsars that were being observed in the centre beam of the receiver. For reference, we include the DMs and RMs for all the relevant pulsars in Table 1. Three of the FRBs have DMs in the top 10 largest values at the time of publication. The four FRB events are shown graphically in Fig. 1. The panels represent the four bursts. The bottom segment of each panel gives the FRB flux density for the total intensity signal (black), linear polarization (red), and circular polarization (blue). The angle of the linear polarization is shown in the upper segment of each panel. The polarization angle of all the FRBs in our sample is flat as a function of time, similar to that of FRB 150807 (Ravi et al. 2016).

FRB 171209 was the first FRB detected as part of the commensal search during PPTA observations. The FRB was detected in one of the outer beams during an observation of PSR J1545-4550. The position of the FRB cannot be well constrained, but the burst originated at a low Galactic latitude of 6.2 . The FRB is relatively wide $(2.5 \mathrm{~ms})$. This width is consistent with that expected from instrumental DM smearing and indeed the preferred model is a DMsmeared impulse with scattering. We obtain an estimate of scattering time-scale to be $0.66 \mathrm{~ms}$. It is the most strongly polarized FRB in our sample. The linear polarization fraction $L_{\mathrm{f}}=1.00 \pm 0.01$, while the fraction of circular polarization is consistent with zero. ${ }^{5}$ We measured the Faraday rotation, which led to a RM value of $121.6 \pm 4.2 \mathrm{rad} \mathrm{m}^{-2}$.

During observations of PSR J2124-3358, we discovered FRB 180309, which is the highest S/N (411) FRB yet detected. It was so bright that the dynamic range of the recorded signal was not sufficient with the cross-products being most affected. The burst is the narrowest in our sample, with a full width at halfmaximum (FWHM) of $0.475 \mathrm{~ms}$ consistent with DM smearing of

\footnotetext{
${ }^{5}$ The polarization degrees are nominal values as reported by the PSRSTAT
} tool, which is part of the PSRCHIVE software suite. 

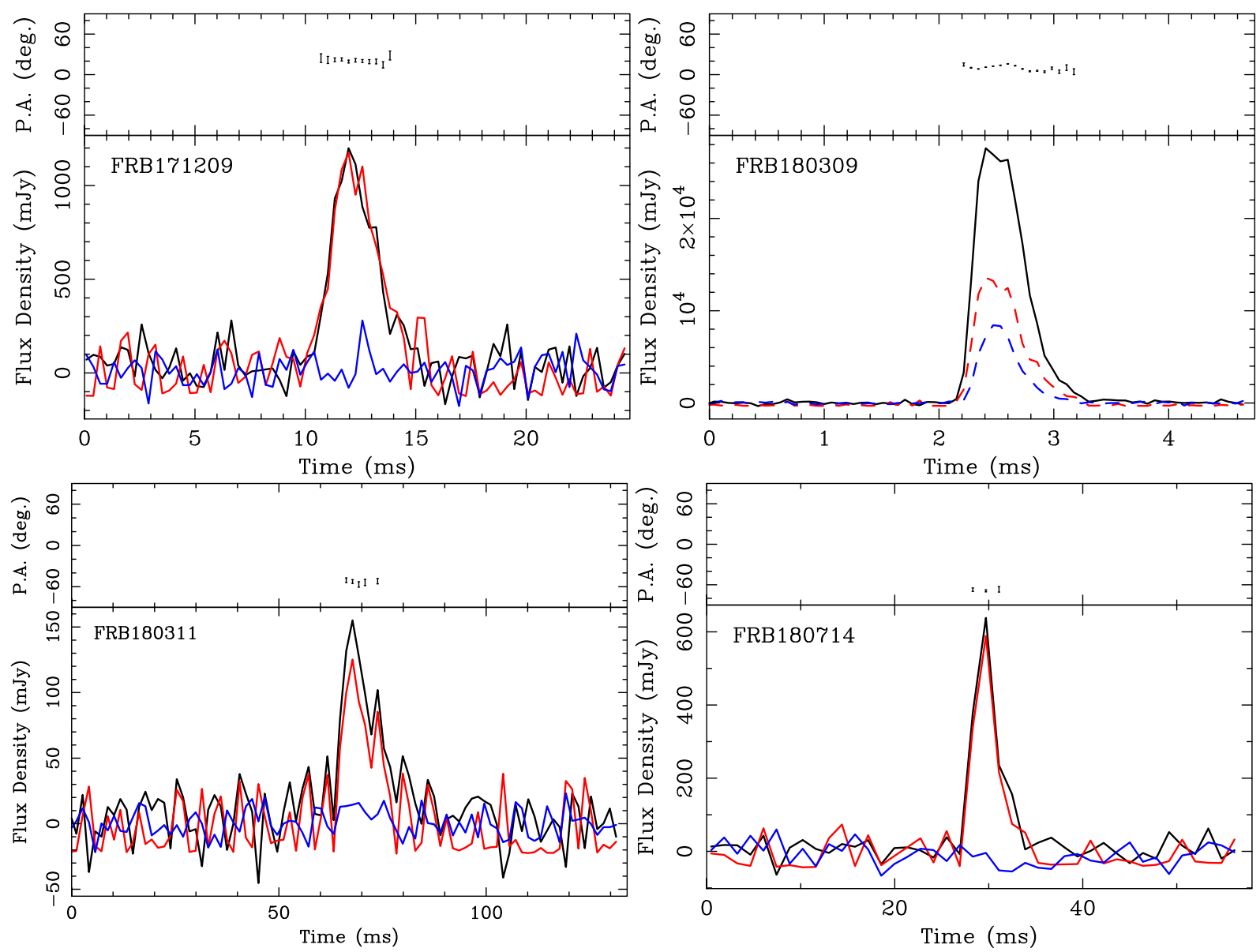

Figure 1. The four subfigures show the polarization position angles (top panels) and polarized pulse profiles (bottom panels, where the black line denotes total intensity, while red and blue show the linear and circular polarization, respectively) for all the four FRBs discovered during PPTA observations. The dashed lines for FRB 180309 indicate that caution is needed when interpreting the polarization.

an unresolved impulse. This narrow width translates into a relatively low estimate of the lower limit of fluence of $13.12 \mathrm{Jy}$ ms. This burst was clearly detected in all beams of the receiver, except for beams 3,4 , and 5 (with a marginal detection in beams 3 and 5), with the highest $\mathrm{S} / \mathrm{N}$ in the central beam of the receiver. After polarization calibration of the data, we estimated the linear polarization fraction $L_{\mathrm{f}}=0.4556 \pm 0.0006$, while circular polarization fraction is lower at $V_{\mathrm{f}}=0.2433 \pm 0.0005$. While the polarimetry was affected by the saturation, we have confirmed the degree of polarization and the spectral structure using other beams where the FRB was not as bright. We note that the Stokes $Q$ was least affected by saturation and remains positive throughout the whole band. From this, we estimated that the modulus of the rotation measure must be less than $\propto 150 \mathrm{rad} \mathrm{m}^{-2}$.

During the same observing session as FRB 180309, we also observed a low S/N burst during an observation of PSR J2129-5721. This burst, FRB 180311, is the widest of our sample with an FWHM of $13.4 \mathrm{~ms}$, and the only burst for which we were able to determine the intrinsic width of $3.8 \mathrm{~ms}$ in addition to a smearing and scattering. Because of its high DM $\left(1570.9 \mathrm{~cm}^{-3} \mathrm{pc}\right)$ and predicted low Galactic contribution to the total DM of $32 \mathrm{~cm}^{-3} \mathrm{pc}$, the inferred redshift is $\approx 2.0$. Despite the high degree of linear polarization $\left(L_{\mathrm{f}}=\right.$ $0.75 \pm 0.03)$ the rotation measure value of $4.8 \pm 7.3 \mathrm{rad} \mathrm{m}^{-2}$ is consistent with zero. The circular polarization fraction is low, but detectable at $V_{\mathrm{f}}=0.11 \pm 0.02$.
Our fourth and (so far) final burst, FRB 180714, was discovered during an observation of PSR J1744-1134. This FRB was detected with an S/N of 22 and a dispersion measure of $1467.92 \mathrm{~cm}^{-3} \mathrm{pc}$. Like FRB 171209 , the burst is very strongly linearly polarized $\left(L_{\mathrm{f}}=\right.$ $0.91 \pm 0.03)$ with a hint of circular polarisation $\left(V_{\mathrm{f}}=0.05 \pm 0.02\right)$ after correcting for the measured $\mathrm{RM}$ of $-25.9 \pm 5.9 \mathrm{rad} \mathrm{m}^{-2}$.

\section{DISCUSSION}

As some FRBs have now been seen to repeat and others detected in the far field of an interferometer system (Caleb et al. 2017), it is clear that at least most FRBs are celestial sources, while numerous arguments point to them as extragalactic pulses. However, the 'perytons' (Burke-Spolaor et al. 2011) that were linked to a microwave oven on the Parkes Observatory site (Petroff et al. 2015b) also highlight that terrestrial signals can produce signals that mimic high-dispersion bursts. However, due to their near-field origin, perytons are detected in all receiver beams simultaneously, and due to their non-dispersive nature, their spectra show deviations from dispersive sweeps. For three of the four FRBs described here, the burst was only detected in a single beam. The brightest FRB was detected in 10 beams at high significance, which is expected for a very intense far-field source given that each beam's sensitivity pattern overlaps with adjacent beams, i.e. while perytons and other near-field detections appear at roughly equal power in all beams, 


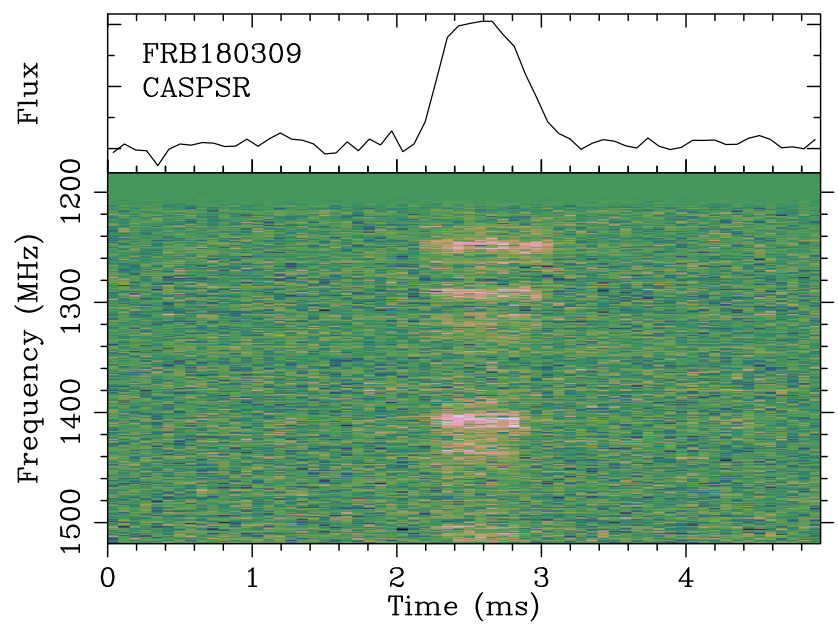

Figure 2. The detection of FRB 180309 in folded pulsar data. The top panel shows the total intensity profile, while the bottom panel shows the spectrum. We note that this spectrum looks very different to the spectrum detected in the BPSR as it was much less saturated.

this source did not. A consistent solution for the position of the burst based on the method of Ravi et al. (2016) will be published elsewhere (Aggarwal et al., in preparation). Regardless, we have searched for any event that may have occurred on the Parkes site and identified that the pressure in a compressor system, one of hundreds of monitoring points, had a step change coincident with the FRB 180309 event within the $10 \mathrm{~s}$ sampling time of the monitoring system. We have tested various scenarios in which we reproduced the spike in pressure without any impact on the observed transient effects and conclude this is most likely just a coincidence.

\subsection{The bright burst (FRB 180309)}

FRB 180309 is the strongest FRB yet detected with the Parkes telescope with the detection $\mathrm{S} / \mathrm{N}$ of 411 . Unfortunately, the event was so bright that it saturated the digitizer system for the multibeam recording, and thus its observed intensity was truncated. As it was discovered in the central beam of the multibeam receiver, we also can study the FRB using the backend instruments that are used to fold the pulsar signal. Here, we present data from CASPSR, which was used to fold and coherently dedisperse the $5 \mathrm{~ms}$ pulsar PSR J2124-3358 at which the telescope was pointed. We dedispersed $8 \mathrm{~s}$ of the data that were detected and averaged at the period of the pulsar at the DM of the FRB. The results of this process are presented in Fig. 2, with the top panel showing the total flux density pulse profile of the burst, while the bottom panel shows the spectrum of the burst. ${ }^{6}$ The $\mathrm{S} / \mathrm{N}$ of the burst in these folded data is 46.2. After taking the integration of $8 \mathrm{~s}$ of data and pulsar period and extra smearing due to CASPSRs channelization being twice as coarse into account, we estimate the intrinsic $\mathrm{S} / \mathrm{N}$ of the burst must have been at least 2616 if the data had not been averaged over the pulsar's multiple rotations. This implies the fluence is underestimated by a factor of 6.4 or more, yielding an estimated adjusted fluence limit $F_{\text {adj }}>83.5 \mathrm{Jy} \mathrm{ms}$.

The estimate of adjusted fluence is well above the fluence limit for the FRB searches with Australian Square Kilometre Array

${ }^{6}$ We note that this spectrum is consistent with the spectrum of the burst in the non-central beams of the multibeam receiver.

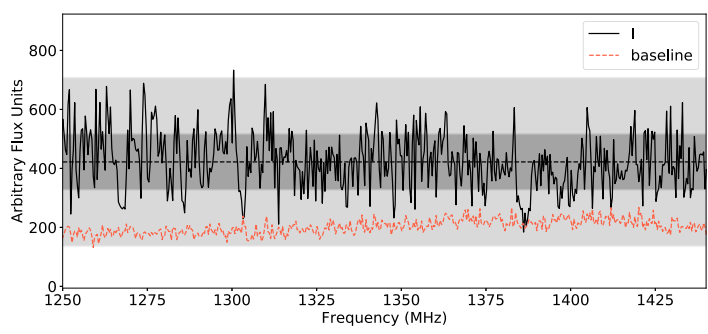

Figure 3. The time-averaged spectrum during FRB 180309 event. The red line is the baseline spectrum of the observed pulsar (PKS J2124-3358), the dark and light grey bounding boxes signify $1 \sigma$ and $3 \sigma \mathrm{rms}$ noise, respectively.

Pathfinder (ASKAP) of $26 \mathrm{Jy} \mathrm{ms}$ for $1 \mathrm{~ms}$ bursts (Shannon et al. 2018) that discovered more than 20 bursts. The bursts observed by ASKAP have strongly modulated spectra, much more so than the population of FRBs typically detected at Parkes, with FRB 150807 (Ravi et al. 2016) and FRB 180301 (Price et al. 2019) being one of a few exceptions among the population of bursts discovered at Parkes. However, note that the modulation of the Parkes-discovered population has not been yet studied in detail. ${ }^{7}$ Macquart et al. (2019) quantified the spectral properties of ASKAP bursts and argued that their modulation is likely to be a propagation effect, further corroborated by lack of such modulation in most Parkes bursts. We find that not only are the spectral properties of FRB $180309^{8}$ similar to the ASKAP bursts, but so are its other properties: DM, width, and fluence, indicating it is a part of the same population as the bursts discussed in Shannon et al. (2018) and Macquart et al. (2019).

As the FRB 180309 was detected in channelized data in the $20-\mathrm{cm}$ observing band, we were able to search for evidence in the spectrum that could relate to $\mathrm{H}$ I absorption in the redshift range spanning $0 \leq$ $z \leq 0.2$, neatly matching the predicted redshift for FRB 180309 of $z \leq 0.19$. Note that, as described by Fender \& Oosterloo (2015), we do not expect a detection of $\mathrm{H}$ I absorption towards Parkes-detected FRBs; however, given the unusually high S/N of FRB 180309, we searched for $\mathrm{H}$ I absorption for this burst. A successful detection of the absorption would provide a lower limit on the redshift of the host galaxy. Fig. 3 shows the time-averaged spectrum of FRB 180309. The black dashed line indicates the mean with the dark and light grey regions signifying $1 \sigma$ and $3 \sigma$ deviations, respectively. The most prominent (but not statistically significant) 'absorption feature' is centred at $1386 \mathrm{MHz}$ and has a frequency FWHM of $\sim 4 \mathrm{MHz}$; at an implied redshift of $z=0.025$, this corresponds to a velocity width of $870 \mathrm{~km} \mathrm{~s}^{-1}$ at FWHM. While astrophysical systems have been found to have similarly high velocity widths (e.g. Morganti, Tadhunter \& Oosterloo 2005), these systems typically have low peak optical depths and are often associated with fast outflows or active galactic nuclei (AGNs) feedback. Given the broad velocity width and low significance of this feature, we conclude that it is unlikely to be associated with a real absorber along the line of sight. We note that the feature is unlikely to be due to the saturation of the BPSR spectrum as it does not coincide with the brightest parts of the spectrum from other beams and CASPSR.

${ }^{7}$ We note Farah et al. (2018) presented highly modulated emission of FRB 170827 detected with the Molonglo Synthesis Telescope.

${ }^{8}$ We note that the scintillation time-scale is consistent with the scintillation seen in the saturated spectrum in the primary beam. The spectrum in the beams with lower $\mathrm{S} / \mathrm{N}$ of the burst shows scintillation on a different scale that is unlikely to be a propagation effect. 
Soon after the detection of this bright burst, we performed follow-up observations using the Australia Telescope Compact Array (ATCA) and the Very Large Array (VLA) interferometers, in addition to imaging of the field with Gemini South telescope. This follow-up, and the potentially related members in the field, will be discussed in a separate publication (Aggarwal et al., in preparation).

Data with the ATCA were recorded in both the continuum and zoom modes at centre frequencies of 2100 and $1386 \mathrm{MHz}$, respectively. The final data sets reached an $\mathrm{rms}$ of $35 \mu \mathrm{Jy}_{\text {beam }}^{-1}$ in continuum and $12 \mathrm{mJy} \mathrm{beam}^{-1}$ for the zoom mode data. The follow-up with the ATCA has contributed to the considerations of the H I absorption above, in that we were unable to detect either a continuum or a H I counterpart at the redshift indicated by the spectral feature discussed in the previous paragraph.

The Neil Gehrels Swift Observatory (Swift; Gehrels et al. 2004) was considered for rapid follow-up in the optical, ultraviolet, and at high energy. However, the target was located only $38^{\circ}$ away from the Sun, thus too close to the Sun to be observed. Observations with Swift would have become possible starting on 2018-02-18, about $11 \mathrm{~d}$ after the FRB detection. We refrained from performing such late-time observations, already attempted in several other FRB follow-ups (e.g. Petroff et al. 2015a, 2017) that were unsuccessful.

\subsection{Implications of the FRB polarimetry}

So far, polarization has been measured for only eight FRBs, of which five have measured RMs, two with no measurement, and one with an RM estimate consistent with zero (see overview by Caleb et al. 2018; Price et al. 2019 for discussion of unusual polarization of FRB 180301). Of the eight FRBs, three have a very high polarization degree ( $>80$ per cent), including the first repeating FRB (Michilli et al. 2018). The latter also has the highest RM measured, with the value changing in time but of the order of $10^{5} \mathrm{rad} \mathrm{m}^{-2}$.

In contrast to majority of non-repeating FRBs, the bursts in our sample are highly polarized, suggesting that strong magnetic fields are involved in their emission mechanism, and show a variety of RMs, which in turn provides insight into strength and structure of magnetic fields in the intergalactic medium. Measurement of polarization of FRBs is important to help understand the emission mechanism (e.g. Houde et al. 2019; Lu, Kumar \& Narayan 2019). Some of the proposed models, such as those proposed by Lyubarsky (2014), Beloborodov (2017), Ghisellini (2017), and Waxman (2017), would need to be adjusted to reproduce the high degrees of polarization observed in a growing number of FRBs.

While we cannot draw definitive conclusions from the polarimetry of just one repeating FRB, it is worth noting that the non-repeating FRBs have different polarization properties to FRB 121102. While all are highly polarized, the RM values are different for all four FRBs, while remaining in range comparable to that of radio pulsars, in contrast to the RM of FRB 121102 that is very large, of the order of $10^{5} \mathrm{rad} \mathrm{m}^{-2}$ and appears to evolve in time (Gajjar et al. 2018; Michilli et al. 2018). Whether this implies a different environment or progenitor remains unclear.

\subsection{Updated FRB event rates}

To date, FRBs discovered at Parkes using the BPSR instrument remain the most uniform sample of FRBs, although we do anticipate the Canadian Hydrogen Intensity Mapping Experiment (CHIME) to discover soon a much larger number of FRBs based on their detection rates from early observations (CHIME/FRB Collaboration et al. 2019a). Having a uniform sample of FRBs is important to
Table 3. Time on sky in the three latitude bins for our survey and the results from Bhandari et al. (2018). The FRB sky rates for respective latitude bins are quoted with 95 per cent confidence.

\begin{tabular}{lccccc}
\hline $\begin{array}{l}\text { Galactic latitude } \\
|b|\end{array}$ & $\begin{array}{c}\text { Previous } \\
\text { searches } \\
(\mathrm{h})\end{array}$ & $\begin{array}{c}\text { PPTA } \\
\text { time } \\
(\mathrm{h})\end{array}$ & $\begin{array}{c}\text { Total } \\
\text { time } \\
(\mathrm{h})\end{array}$ & $\begin{array}{c}N_{\mathrm{FRBs}} \\
(\mathrm{h})\end{array}$ & FRBs sky $^{-1} \mathrm{~d}^{-1}$ \\
\hline$|b| \leq 19.5$ & 3024 & 281 & 3305 & 6 & $3.3_{-1.9}^{+4} \times 10^{3}$ \\
$19.5<|b|<42^{\circ}$ & 2245 & 197 & 2442 & 6 & $4.4_{-2.5}^{+4.4} \times 10^{3}$ \\
$42^{\circ} \leq|b| \leq 90^{\circ}$ & 2088 & 155 & 2243 & 11 & $8.9_{-3.4}^{+5.4} \times 10^{3}$ \\
\hline
\end{tabular}

finally resolve the outstanding issue of Galactic latitude dependence of FRB rates. The rates can also provide insight into the nature of the progenitors (Nicholl et al. 2017; Cao, Yu \& Zhou 2018).

Discussion of the Galactic latitude dependence dates back to some of the first work on FRBs. The discovery of four FRBs at high Galactic latitudes by Thornton et al. (2013) radically increased the number of known FRBs. Soon after, Petroff et al. (2014) searched medium-latitude data from the High Time Resolution Universe Pulsar Survey, which was at lower latitudes and concluded that FRBs are found preferentially at the higher latitudes, providing further support for their extragalactic origin. Burke-Spolaor \& Bannister (2014) arrived at a similar conclusion by searching archival data from Parkes surveys. Macquart \& Johnston (2015) suggested this may be due to scintillation boosting the detection rate at higher latitudes.

Recently, Bhandari et al. (2018) revisited this issue while presenting results from a large amount of time on the sky at the Parkes telescope. The authors found that the discrepancy in rates at different latitudes persisted with the newly released data but has been reduced to lower significance. Here, we repeat their analysis but add 633 more hours of observations and four more bursts, which represents an increase of only 8 per cent of time on the sky but our relatively high rate corresponds to increasing the number of bursts considered by 21 per cent.

Given that our detection pipeline is nearly identical to that of the SUPERB project, we assume we can directly combine our results with those presented in Bhandari et al. (2018). Furthermore, two of our FRBs, FRB 171209 and FRB 180714, were discovered at low Galactic latitudes. Table 3, similar to table 5 of Bhandari et al. (2018), shows the total amount of time and FRBs per latitude bin, as well as the inferred FRB rates above the limiting fluence of $2 \mathrm{Jy} \mathrm{ms.}$ The combined rates are consistent with the previous estimates of the aforementioned authors.

\subsection{Limit on the presence of repeating FRBs}

The non-observation of repeating FRBs in PPTA observations allows limits to be set on their volumetric density (James 2019). Here, we consider limits only on repeating FRBs with properties similar to the most studied repeater, FRB 121102: a power-law distribution of burst indices, with rate $R_{0}=7.4 \mathrm{~d}^{-1}$ above an energy of $E_{0}=1.7 \times 10^{38} \mathrm{erg}$, and rate decreasing with energy to the power of $\gamma=-0.9$.

The FRB detection threshold to a nominal $1 \mathrm{~ms}$ burst is $0.5 \mathrm{Jy} \mathrm{ms}$. To model the effects of the beam shape, we use the simulation of K. Bannister, as per Ravi et al. (2016). Since the rotation angle of the multibeam receiver about the pointing position was kept fixed during PPTA observations, we calculate the mean value of beam sensitivity pattern $B^{-\gamma}$ for each offset angle, which gives the 


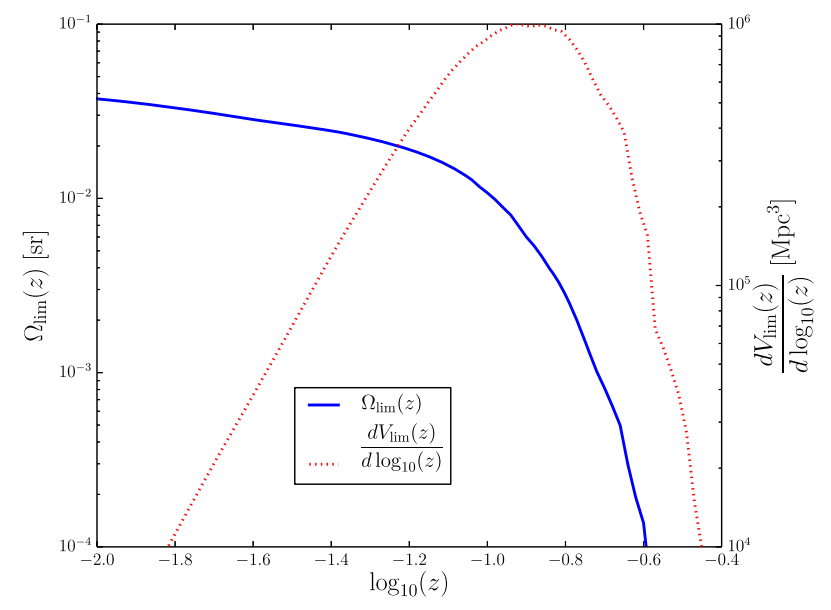

Figure 4. Limits at 95 per cent confidence level on the presence of repeating FRBs from the PPTA observations. Blue solid line: solid angle $\Omega_{\lim }(z)$ over which the presence of any FRBs with properties similar to FRB 121102 can be excluded within redshift $z$. Red dashed line: differential volume at redshift $z$ within which the presence of such an FRB can be excluded.

relative reduction in observed rate to the burst energy distribution with power-law index $\gamma$.

For the observations reported here, each of 24 targets was observed for an average of $26 \mathrm{~h}$, with $61.5 \mathrm{~h}$ on J0437-4715. Following James (2019), the time-on-target and solid angle sensitivity of the beam shape are combined to produce a limiting solid angle, $\Omega_{\lim }(z)$. This gives the solid angle over which the presence of a repeating FRB closer than redshift $z$ with the above properties can be excluded at 95 per cent confidence. This is shown in Fig. 4 (blue solid line). Converting this to a differential volume - Fig. 4, red dotted line - and integrating produces a limiting volume $V_{\lim }$ within which the presence of such an FRB can be excluded. In this case, $V_{\text {lim }}=5 \times 10^{5} \mathrm{Mpc}^{3}$.

This value is much less than the ASKAP/CRAFT lat50 result of $8.4 \times 10^{6} \mathrm{Mpc}^{3}$ for this scenario (James 2019). The orderof-magnitude sensitivity increase of the Parkes observations is largely offset by the reduction in total observing time per pointing comparing to the lat50 survey, while ASKAP's wider field of view produces a much stronger limit. Continued observations of the same fields, however, will allow Parkes to probe higher redshift values than ASKAP. Limits from much longer FRB surveys with Parkes - e.g. SUPERB and HTRU - may not be as strong as these PPTA limits, due to observation time being spread over many pointings.

\section{OUTLOOK}

The FRB discoveries reported here demonstrate the value of commensal observing projects. However, the Parkes receiver suite was recently upgraded and an ultrawide bandwidth (UWL) receiver is commissioned (Hobbs et al., in preparation). The PPTA team will be solely using that new, single-pixel receiver for the majority of future observations.

The backend instrumentation is also being upgraded and will allow commensal high time- and frequency-resolution observing modes along with automatic transient identification. There are advantages and disadvantages for FRB searches with the new receiver. Any FRB detected will be observed over a frequency band between $700 \mathrm{MHz}$ and $4 \mathrm{GHz}$ enabling detailed studies of the spectral index and scintillation properties of any such burst.
With a high-frequency resolution mode, it may also be possible to study HI absorption in the direction of the FRB event in detail. However, having just a single beam has disadvantages. It will be harder to distinguish RFI from astronomical events and any given burst is more likely to be detected in the low-frequency part of the band where the beam is wider implying that any wide-band studies will need to account both for the spectral properties of the FRB, the receiver and the likelihood that the FRB position is offset from the centre of the beam. The event rate will also be lower. The beamwidth in the low part of the band is twice that of the central beam of the multibeam and assuming the amount of time per semester with this receiver will be twice as large as it was with the multibeam. However, we only will have one beam instead of 13 , and ignoring complications due to spectral properties of FRBs, we can expect about two FRB events per semester. We assumed values of a typical observing semester in which we obtain $500 \mathrm{~h}$ of telescope time and that real-time commensal searching is possible with UWL. We note the impact of having only a single beam available for confirming astrophysical origin of any burst is difficult to incorporate in any such estimation.

With new FRBs likely detected with the UWL receiver at Parkes, more with the multibeam observations as part of the Breakthrough Listen (Price et al. 2019) and SUPERB observations, and further searches through archival Parkes data (e.g. Zhang et al. 2019), we expect that the Parkes telescope will continue to increase the known population of FRBs albeit with limited localization potential.

\section{ACKNOWLEDGEMENTS}

The Parkes radio telescope is part of the Australia Telescope, which is funded by the Commonwealth of Australia for operation as a National Facility managed by the Commonwealth Scientific and Industrial Research Organisation (CSIRO). This paper includes archived data obtained through the CSIRO Data Access Portal (http: //data.csiro.au). The authors would like to thank Chris Flynn and Shivani Bhandari for helpful discussions. We are grateful to Amy Lien, Jeff Cooke, and Nicolas Tejos for help with considerations of rapid follow-up. Bill Coles was one of the early proponents of conducting this experiment during the PPTA observations and we thank him for the helpful discussions about the manuscript. We thank the referee for useful comments on the manuscript.

SO, MB, and RS acknowledge Australian Research Council grant FL150100148. MB and RMS acknowledge Australian Research Council grant CE170400001. J-BW is supported by the Youth Innovation Promotion Association of Chinese Academy of Sciences. Work at NRL is supported by NASA. This research has made use of NASA's Astrophysics Data System.

\section{REFERENCES}

Barsdell B. R., 2012, PhD thesis, Swinburne University of Technology Beloborodov A. M., 2017, ApJ, 843, L26

Bhandari S. et al., 2018, MNRAS, 475, 1427

Burke-Spolaor S., Bannister K. W., 2014, ApJ, 792, 19

Burke-Spolaor S., Bailes M., Ekers R., Macquart J.-P., Crawford F. III, 2011, ApJ, 727, 18

Caleb M. et al., 2017, MNRAS, 468, 3746

Caleb M. et al., 2018, MNRAS, 478, 2046

Cao X.-F., Yu Y.-W., Zhou X., 2018, ApJ, 858, 89

Champion D. J. et al., 2010, ApJ, 720, L201

Chatterjee S. et al., 2017, Nature, 541, 58

CHIME/FRB Collaboration et al., 2019a, Nature, 566, 230

CHIME/FRB Collaboration et al., 2019b, Nature, 566, 235 
Dai S. et al., 2015, MNRAS, 449, 3223

Farah W. et al., 2018, MNRAS, 478, 1209

Fender R., Oosterloo T., 2015, MNRAS, 451, L75

Fialkov A., Loeb A., 2016, J. Cosmol. Astropart. Phys., 05, 004

Gajjar V. et al., 2018, ApJ, 863, 2

Gehrels N. et al., 2004, ApJ, 611, 1005

Ghisellini G., 2017, MNRAS, 465, L30

Granet C. et al., 2005, IEEE Antennas Propagation Magazine, 47, 13

Han J. L., Manchester R. N., Lyne A. G., Qiao G. J., van Straten W., 2006, ApJ, 642, 868

Hobbs G. et al., 2012, MNRAS, 427, 2780

Hotan A. W., van Straten W., Manchester R. N., 2004, Publ. Astron. Soc. Aust., 21, 302

Houde M., Rajabi F., Gaensler B. M., Mathews A., Tranchant V., 2019, MNRAS, 482, 5492

James C. W., 2019, MNRAS, 486, 5934

Jaroszynski M., 2019, MNRAS, 484, 1637

Keane E. F. et al., 2018, MNRAS, 473, 116

Keith M. J. et al., 2010, MNRAS, 409, 619

Lorimer D. R., Bailes M., McLaughlin M. A., Narkevic D. J., Crawford F., 2007, Science, 318, 777

Lu W., Kumar P., Narayan R., 2019, MNRAS, 483, 359

Lyubarsky Y., 2014, MNRAS, 442, L9

Macquart J.-P., Johnston S., 2015, MNRAS, 451, 3278

Macquart J. P., Shannon R. M., Bannister K. W., James C. W., Ekers R. D., Bunton J. D., 2019, ApJ, 872, L19

McQuinn M., 2014, ApJ, 780, L33

Manchester R. N., Hobbs G. B., Teoh A., Hobbs M., 2005, AJ, 129, 1993

Manchester R. N. et al., 2013, Publ. Astron. Soc. Aust., 30, 17

Michilli D. et al., 2018, Nature, 553, 182

Morganti R., Tadhunter C. N., Oosterloo T. A., 2005, A\&A, 444, L9

Nicholl M., Williams P. K. G., Berger E., Villar V. A., Alexander K. D., Eftekhari T., Metzger B. D., 2017, ApJ, 843, 84
Osłowski S. et al., 2018a, Parkes Observations of Fast Radio Bursts FRB 171209, FRB 180309, FRB 180311 and FRB 180714. Available at: https: //doi.org/10.25919/5cb0344970ef3

Osłowski S. et al., 2018b, Astron. Telegram, 11385, 1

Osłowski S. et al., 2018c, Astron. Telegram, 11396, 1

Osłowski S. et al., 2018d, Astron. Telegram, 11851, 1

Petroff E. et al., 2014, ApJ, 789, L26

Petroff E. et al., 2015a, MNRAS, 447, 246

Petroff E. et al., 2015b, MNRAS, 451, 3933

Petroff E. et al., 2016, Publ. Astron. Soc. Aust., 33, e045

Petroff E. et al., 2017, MNRAS, 469, 4465

Price D. C. et al., 2019, MNRAS, 486, 3636

Prochaska J. X., Zheng Y., 2019, MNRAS, 485, 648

Ravi V., 2019, MNRAS, 482, 1966

Ravi V. et al., 2016, Science, 354, 1249

Ravi V. et al., 2019, BAAS, 51, 420

Schwarz G., 1978, Ann. Stat., 6, 461

Shannon R. M. et al., 2015, Science, 349, 1522

Shannon R. M. et al., 2017, Astron. Telegram, 11046, 1

Shannon R. M. et al., 2018, Nature, 562, 386

Spitler L. G. et al., 2016, Nature, 531, 202

Staveley-Smith L. et al., 1996, Publ. Astron. Soc. Aust., 13, 243

Tendulkar S. P. et al., 2017, ApJ, 834, L7

Thornton D. et al., 2013, Science, 341, 53

van Straten W., Demorest P., Osłowski S., 2012, Astron. Res. Technol., 9, 237

Waxman E., 2017, ApJ, 842, 34

Yao J. M., Manchester R. N., Wang N., 2017, ApJ, 835, 29

Zhang S. B., Hobbs G., Dai S., Toomey L., Staveley-Smith L., Russell C. J., Wu X. F., 2019, MNRAS, 484, L147

This paper has been typeset from a $\mathrm{T}_{\mathrm{E}} \mathrm{X} / \mathrm{LT} \mathrm{E} \mathrm{X}$ file prepared by the author. 